\title{
Lymph node transplant in Kenya: a case series of 20 patients
}

\author{
AO Wamalwa ${ }^{1}$, FW Nangole ${ }^{2}$, SO Khainga ${ }^{3}$ \\ 1 Plastic Surgery Registrar, School of Medicine, University of Nairobi. \\ 2 Consultant Plastic Surgeon \& Lecturer; Department of Surgery (Plastic), University of Nairobi, Nairobi, Kenya \\ 3 Consultant Plastic Surgeon \& Associate Professor; Department of Surgery (Plastic), University of Nairobi, Nairobi, Kenya
}

Corresponding author: Dr Alex Wamalwa (aleoke@gmail.com)

\begin{abstract}
Summary
Chronic limb lymphedema is a debilitating condition whose definitive management eludes medical practice to date. Lymph node transplant results in improvement of symptomatology amongst patients with lymphedema non-responsive to nonoperative management. A retrospective audit of all patients who underwent lymph node transplant in Nairobi, Kenya for the period June 2014-June 2017 (three years) was done. We report improvement of symptomatology amongst patients with stage II lymphedema non-responsive to non-operative management. We also highlight surgical considerations taken during the management of these cases.
\end{abstract}

\section{Introduction}

Lymphedema results from dysfunctional, non-functional or destroyed lymphatic channels. The chronically heavy extremity impairs utility of the limb. Abnormal accumulation of protein rich interstitial fluid predisposes the patient to recurrent episodes of ulceration, lymphangitis and or cellulitis. Physical therapy and compression therapy are the main nonoperative management modalities to relieve the symptoms. ${ }^{1}$ Lymph node transplant or free vascularized lymph node transfer (VLNT) is the transfer of functional lymph nodes with their blood vessels onto new sites to facilitate neolymphangiogenesis. $^{2}$ Where non-operative management fails, lymph node transplant is reported as a more effective modality compared to lymphaticovenular anastomosis or lymphatic-lymphatic bypass or lymphaticovenous bypass.", ${ }^{4}$ Furthermore, these other physiologic surgical options are quite long and tedious and require supermicrosurgical skills. Surgical excision procedures are reserved for irreversible late stage procedures and often result in poor outcomes..$^{5}$ Lymph node transplant is a promising treatment modality and our retrospective case series adds to the growing evidence of its application. The objective was to evaluate the effectiveness of lymph node transplant on the preoperative symptomatology. This is the first experience reported from Kenya.

\section{Patients and methods}

In our setting, Nairobi, Kenya, microsurgery is a skill only found and practiced by plastic surgeons. For the period of the audit, there were only two operating microsurgeons. The procedure was undertaken in both the public hospital (Kenyatta National Hospital) and private hospitals (the Nairobi Hospital, Nairobi South Hospital, the Aga Khan University Hospital, and the M.P. Shah Hospital).

In this retrospective audit, we included all patients who underwent lymph node transplant for lymphedema during the period of June 2014 to June 2017. Lymphedema was defined as chronic swelling of an extremity confirmed by lymphoscintigraphy and graded according to the International Society of Lymphology (ISL). Lymph node transplant was defined as the transfer of functional lymph nodes utilizing microvascular anastomoses at the recipient site. A two team approach was incorporated: one team harvested the lymph nodes while the other evaluated and prepared the recipient site. Donor lymph nodes were harvested from the submandibular and inguinal lymph node basins for lower limb and upper limb lymphedema recipient sites, respectively. Microvascular anastomosis was done under microsurgical loupes $4.5 \mathrm{x}$ and $5 \mathrm{x}$ using nylon 9-0. Routine irrigation of vessels with heparinized saline solution (5000 units in $500 \mathrm{mls}$ of normal saline) and $20 \%$ lignocaine was done. 5000 units of intravascular heparin units was given to patients where the anastomoses took 


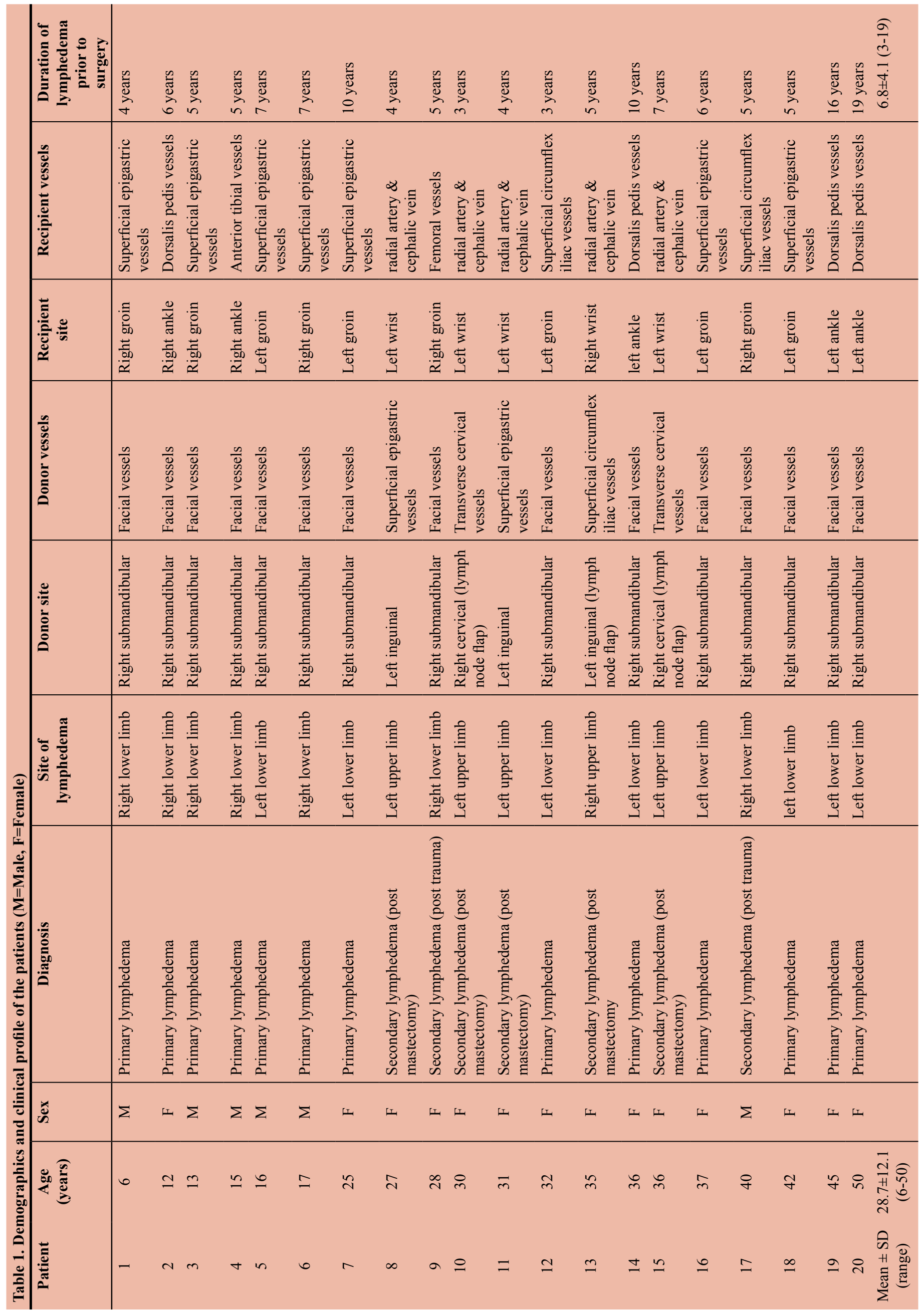


Table 2. Patient outcomes

$\mathrm{S}=$ small, $\mathrm{M}=$ medium, $\mathrm{L}=$ large, $\mathrm{XL}=$ extra-large, $\mathrm{XXL}=$ extra-extra-large; $\mathrm{N} / \mathrm{A}$ - not applicable (symptomatology not present).

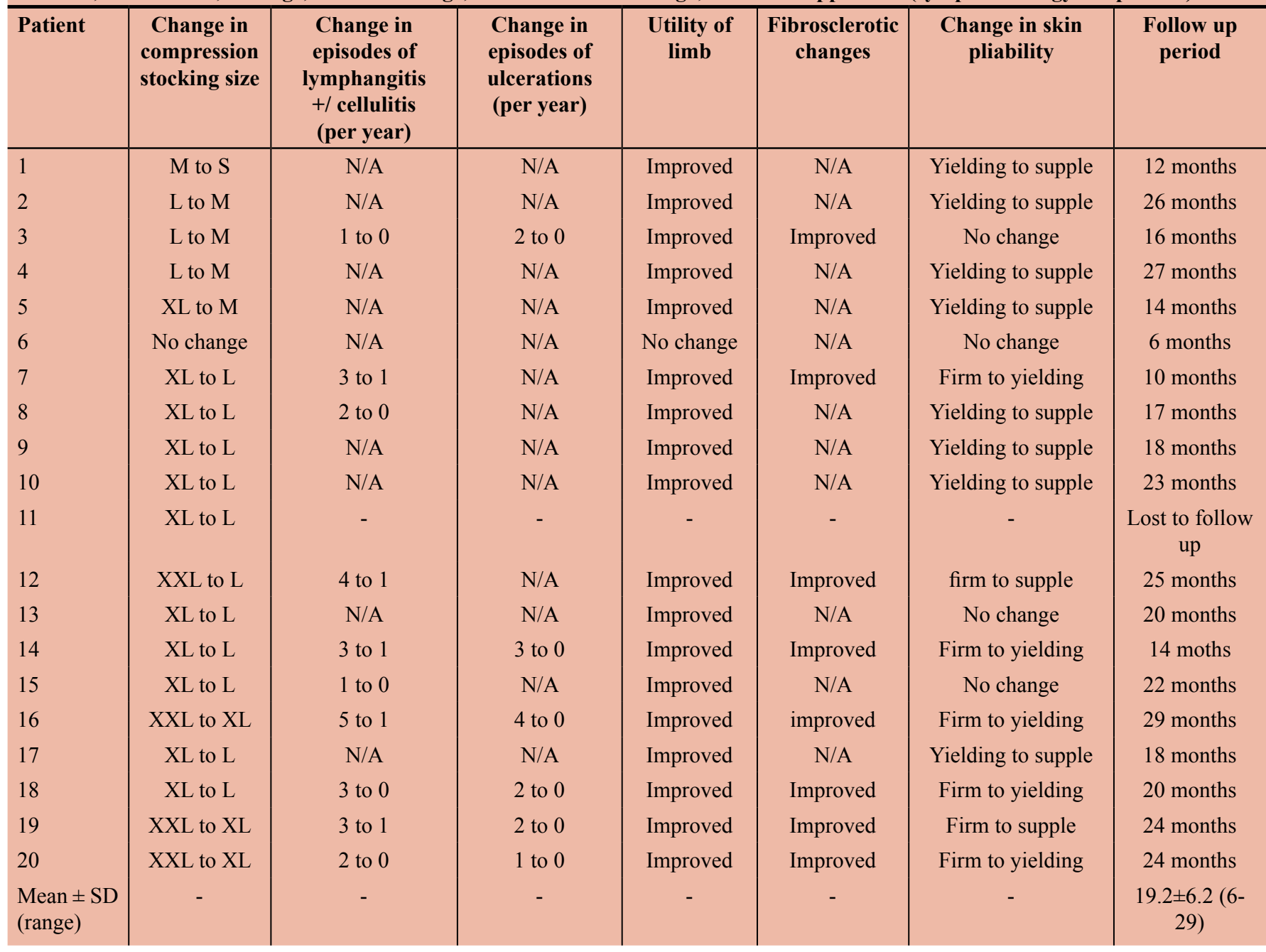

Table 3. Compression stocking size chart

$\mathrm{S}=$ small, $\mathrm{M}=$ medium, $\mathrm{L}=$ =large, $\mathrm{XL}=$ extra-large, $\mathrm{XXL}=$ extra-extra-large

\begin{tabular}{|c|c|c|c|}
\hline \multicolumn{4}{|c|}{ Lower limb stocking size chart } \\
\hline Size & Ankle circumference (cm) & Calf circumference $(\mathrm{cm})$ & Thigh circumference (cm) \\
\hline $\mathrm{S}$ & $19-22$ & $28-34$ & $42-57$ \\
\hline M & $22-24$ & $32-38$ & $48-64$ \\
\hline $\mathrm{L}$ & $25-27$ & $36-42$ & $54-71$ \\
\hline $\mathrm{XL}$ & $28-30$ & $40-46$ & $60-78$ \\
\hline XXL & $30-32$ & $42-50$ & $65-85$ \\
\hline \multicolumn{4}{|c|}{ Upper limb stocking size chart } \\
\hline Size & Wrist circumference $(\mathrm{cm})$ & $\begin{array}{l}\text { Mid-forearm circumference } \\
(\mathrm{cm})\end{array}$ & Mid-arm circumference $(\mathrm{cm})$ \\
\hline $\mathrm{S}$ & $14-20$ & $18-23$ & $22-40$ \\
\hline M & $16-22$ & $23-29$ & $27-45$ \\
\hline $\mathrm{L}$ & $18-24$ & $29-34$ & $33-51$ \\
\hline
\end{tabular}




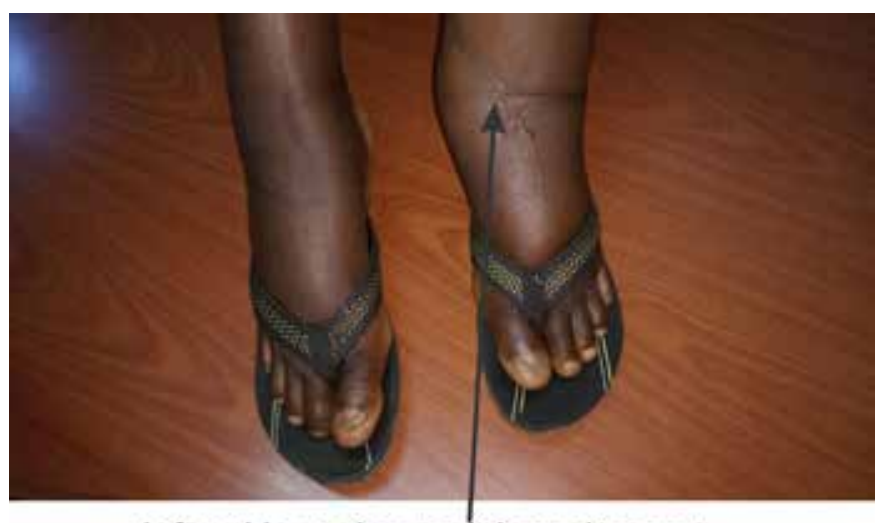

left ankle region recipient site scar

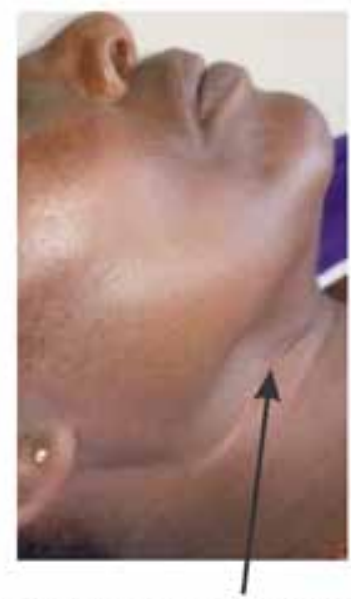

right submandibular region donor site scar

Figure 1. Post-operative healed sites of donor right submandibular region and recipient left ankle region

longer than anticipated. No other adjunct procedures were performed. Postoperative protocol included antimicrobials (amoxicillin/clavulanate $45 \mathrm{mg} / \mathrm{kg} /$ day or ceftriaxone 1-2 g/ day for 1 week plus clindamycin $15 \mathrm{mg} / \mathrm{kg} /$ day for 2 weeks), analgesics (paracetamol $45 \mathrm{mg} / \mathrm{kg} /$ day, diclofenac $2 \mathrm{mg} / \mathrm{kg}$ / day plus an opioid), enoxaparin $(0.5 \mathrm{mg} / \mathrm{kg} /$ day $)$ for 5 days, low dose aspirin $(4 \mathrm{mg} / \mathrm{kg} /$ day) for 2 weeks, limb elevation for 3 months and postoperative compression therapy (started 2 weeks post-surgery and continued for one year). The compression stockings were changed every 3 months. In patients with ulcerations, lymphangitis and or cellulitis, surgery was performed after treatment and resolution of the infection and healing of the wound.

Data was collected from patient medical records perioperative and review consultation notes, operating theatre notes, treatment prescriptions and radiology results. Data recorded included patient demographics, length of symptoms and postoperative outcomes (change in limb size, change in skin pliability, fibrosclerotic skin changes and change in episodes per year of ulcerations and lymphangitis/cellulitis, improvement in limb utility) and the perioperative surgical protocol. Change in limb size was determined by change in stocking size. Skin pliability tactile assessment was a modification of the parameter from the Vancouver Scar scale: normal, supple, yielding, firm (in order of reducing pliability).

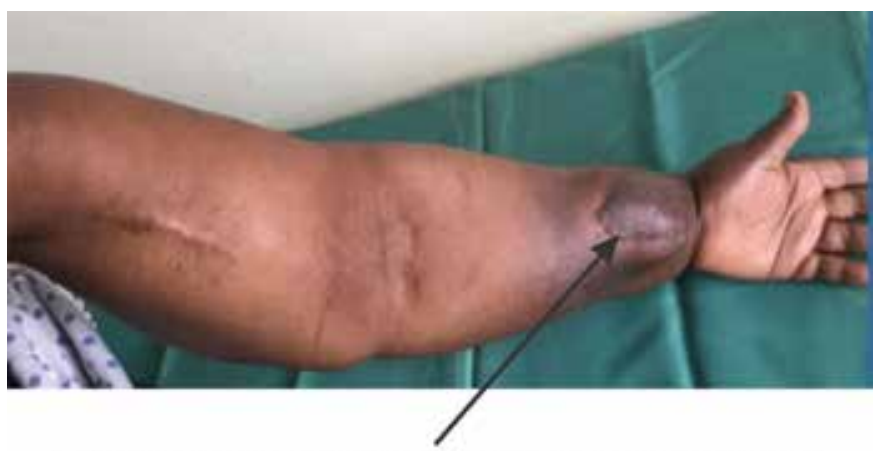

left wrist recipient lymph node flap healed scar

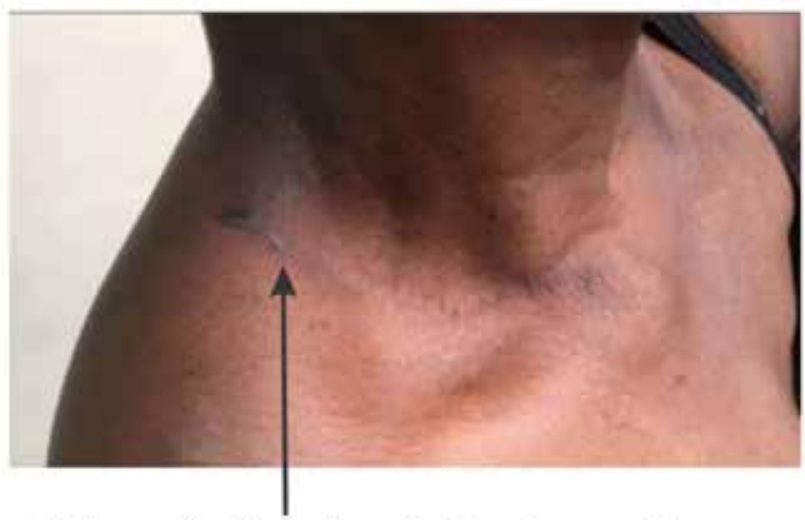

right cervical lymph node flap donor site scar

Figure 2. Post-operative healed sites of donor right cervical region and recipient left wrist region in lymph node flap

Limb utility was subjective report given by the patient during reviews (either improved or not improved). Fibrosclerotic skin changes was determined and assessed by the clinician. Perioperative reviews was done by the operating surgeon. Data was analyzed using descriptive statistics; the mean was calculated. Follow up period was for three years (still ongoing). Approval was sought and granted by the Kenyatta National Hospital/University of Nairobi Ethics and Research Committee (KNH/UON ERC).

\section{Results}

Twenty patients ( $\mathrm{n}=6$ male, $\mathrm{n}=14$ female; median age: 30.5 years; and range 6 to 50 years) with lymphedema underwent lymph node transplant from the period of June 2014 to June 2017. Six patients underwent the procedure in the public hospital.

Of all the patients $(n=20)$, the majority had primary lymphedema (13 patients) and the lower limb was mainly affected (15 patients) (See Table 1). Mean duration of lymphedema prior to surgery was 6.8 years. One patient was lost to follow-up. The mean duration of follow up was 19.2 months.

Most of the patients had improved utility of the affected limb and improved skin pliability (See Table 2). All the patients experienced reduction in limb size as determined by 
reduction in compression stocking size (See Table 3). Patients with prior episodes of ulcerations and lymphangitis/cellulitis experienced reduction in occurrence.

All patients had an uneventful recovery and wounds healed well (See Figure 1 and 2). There were no complications.

\section{Discussion}

This is the first experience on lymph node transplant from Kenya spanning three years. Patient selection is key to success and the criteria reported was based on the senior surgeon's preference and experience in other free flap procedures.

On-table confirmation of functional arterial and venous anastomoses is essential to avoid "lymph node grafting". Due to the edema and or fibrosclerosis, most of the recipient vessels have weak walls; and it may take quite some time to identify a suitable vein. A clear understanding of the varied vascular anatomy of the limb aids in prompt vessel dissection and identification. Patience is an attribute often tested in such circumstances. At the recipient site, the most suitable vessels (good length, caliber and blood flow) were chosen for anastomosis. We recommend a two-team approach: one for harvesting and another for recipient anastomosis to avoid fatigue and mistakes. We opted for and recommend donor lymph nodes of the neck for relative ease of dissection, large caliber donor vessels and limited donor site complications. ${ }^{6}$ The risk of donor site lymphedema of the neck is also quite low as the neck has a robust lymphatic network. ${ }^{7}$ We report neither donor site nor recipient site complications. Contrary to Lee et al., we had no incidence of donor site lymphedema. ${ }^{8}$ While Nguyen at al. encountered other donor site complications, reports from Saaristo et al. and Viitanen et al. indicated recipient site complications. ${ }^{9-11}$ Comparison to these three studies is not appropriate as their patients underwent simultaneous breast reconstruction with VLNT; a more technically demanding procedure.

Use of enoxaparin was limited to the five days when the patient was on strict bed rest post surgery, following which ambulation was initiated. Anti-platelet use was based also on surgeon experience and preference. Postoperative compression therapy was routinely initiated at two weeks when the anastomosis was expected to have healed/matured. Compression therapy was done both pre and post-surgery; based on surgeons experience it hastens improvement in skin pliability. Patient adherence to rehabilitation protocol and compression therapy is also a significant contributor to success of the procedure. Whereas reports by Granzow et al. and Becker et al. highlight ability to reduce and discontinue postoperative compression therapy, there is no analysis of this ability to lymphedema stage. ${ }^{12,13}$ We opt for long term compression therapy for our stage II lymphedema patients, whose spontaneously irreversible lymphedema often relapsed and retarded any improvement in limb size reduction. ${ }^{14}$

In the earlier years (year 2014-2015), the third author opted for ankle region as the recipient site in lower limb lymphedema. During that period, the groin was utilized only when the limb had severe verrucous changes with notable dilated and weak vessels. A paradigm shift in the latter years has seen the groin recipient as the standard. The lymph node flap was utilized to ensure tension-free closure in a region with significant edema. All the patients were unwilling to undergo a postoperative lymphoscintigraphy due to the high cost.

Lymph node transplant resulted in reduction in limb size and improved utility of the affected limb. Utility of the limb was reported by the patient as improved ability to utilize limb in daily activities. Despite the majority of our patients reporting improved limb utility, we cannot equate this to patient satisfaction. Patient satisfaction, which has been reported to be high post VLNT by Patel et al. and Gharb et al., is a reflection of multiple quality of life factors. ${ }^{15,16}$ Although subjectively assessed, our report of reduction in limb size is concordant to studies by Patel et al. and Chen et al. ${ }^{17,18}$ The only patient reporting no change in limb utility and size has only been reviewed six months post-surgery. All patients with prior episodes of ulceration $(n=6)$, lymphangitis and or cellulitis $(n=10)$ have noted reduction in their occurrence. Studies by Cheng at al. also indicated reduction in frequency of infections post VLNT. ${ }^{19,20}$ We further noted improvement in fibrosclerotic changes. This is the first study to report on positive changes in episodes of ulcerations and fibrosclerotic changes.

Success in management of lymphedema using lymph node transplant has been variously reported and our study affirms the same ${ }^{21-23}$ In our experience, the most dramatic changes that we noted and were appreciated by the patients were the reduction in frequency of ulcerations, lymphangitis and or cellulitis and improvement in fibrosclerotic changes.

\section{Limitations of the Study}

Our study is limited by: small sample size, surgeon bias as there was no independent assessor and inadequate measure of limb utility. However, there are no objective measures of limb utilization that have been validated in assessing lymphedema treatment modalities. We further acknowledge our study's drawback in assessing changes in limb size. Despite having regular changes in stockings, they stretch and are unreliable compared to the standard limb circumference measurement. We also did not record when the patients stopped physiotherapy.

\section{Recommendations}

We recommend identification and validation of functional (limb utility) and quality of life assessment tools to objectively assess lymphedema treatment modalities. For example: the six minute walk test for lower limb, isometric muscle strength for both upper and lower limbs, the 36-Item Short Form Health Survey questionnaire and the WHOQOLBREF questionnaire. ${ }^{24-26}$ We also recommend utilization of photographs to objectively assess changes in skin fibrosclerosis. 


\section{Conclusion}

Based on our small series of patients, lymph node transplant results in improvement of symptomatology amongst patients with stage II lymphedema non-responsive to non-operative management.

\section{Conflict of interest}

None

\section{Funding}

None

\section{REFERENCES}

1. 1. NLN Medical Advisory Committee. The diagnosis and the treatment of lymphedema. In: Position statement of the national lymphedema network. Feb 2011. Available from: http://www. lymphnet.org/pdfDocs/nlntreatment.pdf.

2. 2. Suami H, Scaglioni MF, Dixon KA, Tailor RC. Interaction between vascularized lymph node transfer and recipient lymphatics after lymph node dissection - a pilot study in a canine model. J Surg Res. 2016;204(2):418-27.

3. 3. Ozturk CN, Ozturk C, Glasgow M, Platek M, Ashary $\mathrm{Z}$, Kuhn J, et al. Free vascularized lymph node transfer for treatment of lymphedema: A systematic evidence based review. J Plast Reconstr Aesthet Surg. 2016;69(9):1234-47.

4. 4. Akita S, Mitsukawa N, Kuriyama M, Kubota Y, Hasegawa M, Tokumoto $\mathrm{H}$, et al. Comparison of vascularized supraclavicular lymph node transfer and lymphaticovenular anastomosis for advanced stage lower extremity lymphedema. Ann Plast Surg. 2015;74(5):573-9

5. 5. Tiwari A, Cheng KS, Button M, Myint F, Hamilton G. Differential diagnosis, investigation, and current treatment of lower limb lymphedema. Arch Surg. 2003;138(2):152-61.

6. 6. Scaglioni MF, Arvanitakis M, Chen YC, Giovanoli P, Chia-Shen Yang J, Chang EI. Comprehensive review of vascularized lymph node transfers for lymphedema: Outcomes and complications. Microsurgery. 2018;38(2):222-9.

7. 7. Pan WR, Le Roux CM, Briggs CA. Variations in the lymphatic drainage pattern of the head and neck: further anatomic studies and clinical implications. Plast Reconstr Surg. 2011;127(2):611-20.

8. 8. Lee B, Laredo J, Neville R. Reconstructive surgery for chronic lymphedema: a viable option, but. Vascular. 2011;19(4):195-205.

9. 9. Nguyen AT, Chang EI, Suami H, Chang DW. An algorithmic approach to simultaneous vascularized lymph node transfer with microvascular breast reconstruction. Ann Surg Oncol. 2015;22(9):2919-24.

10. 10. Saaristo AM, Niemi TS, Viitanen TP, Tervala TV, Hartiala P, Suominen EA. Microvascular breast reconstruction and lymph node transfer for postmastectomy lymphedema patients. Ann Surg. 2012;255(3):468-73.

11. 11. Viitanen TP, Visuri MT, Hartiala P, Mäki MT, Seppänen MP, Suominen EA, et al. Lymphatic vessel function and lymphatic growth factor secretion after microvascular lymph node transfer in lymphedema patients. Plast Reconstr Surg Glob Open. 2013;1(2):1-9.
12. 12. Granzow JW, Soderberg JM, Kaji AH, Dauphine C. An effective system of surgical treatment of lymphedema. Ann Surg Oncol. 2014;21(4):1189-94.

13. 13. Becker C, Assouad J, Riquet M, Hidden G. Postmastectomy lymphedema: long-term results following microsurgical lymph node transplantation. Ann Surg. 2006;243(3):313-5.

14. 14. International Society of Lymphology. The diagnosis and treatment of peripheral lymphedema. Consensus document for the International Society of Lymphology. Lymphology. 2003;36(2):84-91.

15. 15. Patel KM, Lin CY, Cheng MH. A prospective evaluation of lymphedema-specific quality-of-life outcomes following vascularized lymph node transfer. Ann Surg Oncol. 2015;22(7):2424-30.

16. 16. Gharb BB, Rampazzo A, Spanio di Spilimbergo S, Xu ES, Chung KP, Chen HC. Vascularized lymph node transfer based on the hilar perforators improves the outcome in upper limb lymphedema. Ann Plast Surg. 2011;67(6):589-93.

17. 17. Patel KM, Lin C-Y, Cheng M-H. From theory to evidence: long term evaluation of the mechanism of action and flap integration of distal vascularized lymph node transfers. J Reconstr Microsurg. 2015;31(1):26-30.

18. 18. Chen $\mathrm{R}, \mathrm{Mu} \mathrm{L}$, Zhang $\mathrm{H}$, Xin $\mathrm{M}$, Luan $\mathrm{J}, \mathrm{Mu} \mathrm{D}$, et al. Simultaneous breast reconstruction and treatment of breast cancer-related upper arm lymphedema with lymphatic lower abdominal flap. Ann Plast Surg. 2014;73(Suppl 1):S12-7.

19. 19. Cheng MH, Huang JJ, Nguyen DH, Saint-Cyr M, Zenn MR, Tan BK, et al. A novel approach to the treatment of lower extremity lymphedema by transferring a vascularized submental lymph node flap to the ankle. Gynecol Oncol. 2012;126(1):938.

20. 20. Cheng MH, Chen SC, Henry SL, Tan BK, Lin MC, Huang JJ. Vascularized groin lymph node flap transfer for postmastectomy upper limb lymphedema: flap anatomy, recipient sites, and outcomes. Plast Reconstr Surg. 2013;131(6):1286-98.

21. 21. Liu HL, Pang SY, Lee CC, Wong MM, Chung HP, Chan YW. Orthotopic transfer of vascularized groin lymph node flap in the treatment of breast cancer-related lymphedema: Clinical results, lymphoscintigraphy findings, and proposed mechanism. J Plast Reconstr Aesthet Surg. 2018;71(7):1033-40.

22. 22. Raju A, Chang DW. Vascularized lymph node transfer for treatment of lymphedema: a comprehensive literature review. Ann Surg. 2015;261(5):1013-23.

23. 23. Batista BN, Germain M, Faria JC, Becker C. Lymph node flap transfer for patients with secondary lower limb lymphedema. Microsurgery. 2015. Available at: http://dx.doi. org/10.1002/micr.22404

24. 24. Montgomery PS, Gardner AW. The clinical utility of a sixminute walk test in peripheral arterial occlusive disease patients. J Am Geriatr Soc. 1998;46:706-11.

25. 25. Saris-Baglama RN, Dewey CJ, Chisholm GB, et al. Quality Metric health outcomes ${ }^{\mathrm{TM}}$ scoring software 4.0. Lincoln, RI: QualityMetric Incorporated. 2010;138.

26. 26. Asnani MR, Lipps GE, Reid ME. Utility of WHOQOLBREF in measuring quality of life in Sickle Cell Disease. Health Qual Life Outcomes. 2009;7:75. 